\title{
Application of the ERK signaling pathway inhibitor PD98059 in long-term in vivo experiments
}

\author{
X.Y. Chen ${ }^{1}$, H.Z. Cai ${ }^{1}$, X.Y. Wang' ${ }^{2}$, Q.Y. Chen' ${ }^{2}$, H. Yang ${ }^{2}$, Y.J. Chen ${ }^{2}$ and Y.P. Tang ${ }^{2}$ \\ ${ }^{1}$ The First Affiliated Hospital of Hunan University of Traditional Chinese Medicine, \\ Changsha, Hunan Province, China \\ ${ }^{2}$ Hunan University of Traditional Chinese Medicine, Changsha, Hunan Province, \\ China
}

Corresponding author: X.Y. Chen

E-mail: chenxinyuchen@163.com

Genet. Mol. Res. 14 (4): 18325-18333 (2015)

Received June 22, 2015

Accepted September 11, 2015

Published December 23, 2015

DOI http://dx.doi.org/10.4238/2015.December.23.20

ABSTRACT. The aim of this study was to explore methods by which the ERK signaling pathway inhibitor PD98059 (PD) could be used in long-term in vivo experiments. Forty healthy New Zealand rabbits were randomly divided into blank control, model control, PD low-dose, PD high-dose, PD blank, dimethyl sulfoxide (DMSO) control, DMSO blank, and positive control groups. The corresponding treatments were administered to each experimental group over the course of four weeks, after which, total ERK1/2 and ERK5 protein levels, protein phosphorylation, and gene expression were measured in myocardial tissues. Treatment of rabbits with Adriamycin (doxorubicin) resulted in the significant overall differences in ERK $1 / 2$ and ERK5 phosphorylation $(P<0.05)$. Compared with the model control group, changes in phosphorylated ERK $1 / 2$ and phosphorylated ERK5 were lowest in the PD high-dose group $(P<0.05)$. No significant differences in total protein and mRNA levels of myocardial ERK $1 / 2$ and ERK5 were detected between the groups after four weeks $(P>0.05)$. Continuous intravenous injection of PD98059 significantly reduced phosphorylation of ERK1/2 
and that of ERK5. In conclusion, Adriamycin-induced myocardiopathy and abnormal ERK signaling might constitute a valuable model foruse in long-term experiments. These methods may provide a theoretical basis for related in vivo studies of long duration.

Key words: PD98059; ERK pathway; Signaling pathways; In vivo experiments

\section{INTRODUCTION}

Mitogen-activated protein kinases (MAPKs) are found widely in mammals, and the MAPK signaling pathway plays an important role in the progression of many diseases. In recent years, numerous primary research efforts have helped to define the structure and activation mechanism of this pathway, and demonstrate its essential function in regulating complex cellular processes such as differentiation, proliferation, and apoptosis in response to extracellular signal-regulated stimulation (Zhang and Liu, 2002).

The extracellular signal-regulated kinases $1 / 2$ (ERK1/2) represent an important subfamily of MAPKs. Upon activation, ERK1/2 phosphorylate various targets, including cytoplasmic and membrane proteins, and transcription factors (Kato et al., 2003). This induces the transcription and translation of primary and secondary response genes, such as in the activation of proto-oncogenes (e.g., c-Fos, c-Jun, and c-Myc), mediating the effects of external stimuli on cell proliferation (Yasaka and Hayashida, 2001). ERK1/2 pathways are involved in many physiological processes, including cell growth, development, differentiation, and proliferation. In particular, previous investigations have demonstrated that their influence on developmental processes is important in pathology research, featuring in studies of cancer and cardiovascular and immune system diseases, and has recently become a popular research topic (Chen et al., 2014; Li et al., 2014; Zhang et al., 2014).

ERK5 was initially described as being a MAPK family member similar to ERK1/2. Localized in the cytoplasm, it receives extracellular signals and is activated by an upstream kinase before being transferred to the nucleus, where it exhibits transcriptional regulatory activity by phosphorylating myocyte enhancer factor 2. Since Zhou et al. (1995) first cloned ERK5 in 1995, several functions previously considered to be performed by ERK1/2 have now been attributed to ERK5. Although the biological role of this protein is largely undefined, it is known to perform essential functions in cardiovascular development, including vascular development and proliferation (Nishimoto and Nishida, 2006), maintenance of vascular integrity, cell growth under hypoxia, and reactive oxygen species and mitogen stimulation. Regan et al. (2002) have provided evidence that ERK5-deficient mice demonstrate retarded extraembryonic blood vessel and cardiovascular system development during the embryonic stage. In addition, Hayashi et al. (2004) showed that ERK5-knockout adult mice die within two to four weeks, reinforcing a potential role for ERK5 in cardiovascular development.

Pathway inhibitors have often been used in the basic study of cell signaling, blocking a pathway of interest to observe changes to some index of effect in experimental procedures. PD98059, a non-adenosine triphosphate competitive MAPK kinase (MEK) inhibitor (Dudley et al., 1995), significantly blocks the ERK pathway. It has been extensively used in studies of ERK signaling, owing to its high selectivity. In contrast, the c-Jun N-terminal kinase (JNK, also known as stress-activated protein kinase or SAPK) and p38 pathways are less sensitive to this compound. Recent studies have revealed that this molecule operates as an effective MEK-specific inhibitor in diverse cell types, resulting in clear changes in downstream protein and inflammatory factors 
(Gregg and Fraizer, 2011; Yuan et al., 2011). To date, PD98059 has frequently been utilized in short-term evaluations of in vitro and in vivo experiments, but rarely in long-term in vivo trials involving inhibition of the ERK1/2 and ERK5 pathways. In the current study, Adriamycin (doxorubicin)induced myocardiopathy was used as an abnormal expression model of ERK signaling, with the aim of establishing an appropriate method for the long-term in vivo application of PD98059, providing a theoretical basis for related research.

\section{MATERIAL AND METHODS}

\section{Experimental materials}

\section{Animals}

Forty healthy male New Zealand rabbits, weighing on average $1.85 \pm 0.11 \mathrm{~kg}$, were provided by the Laboratory Animal Center of Hunan University of Traditional Chinese Medicine, experimental animal license number SCXK (Xiang) 2009-0012. Rabbits were kept under controlled conditions, consisting of free access to food and water, a 12:12 h light-dark cycle (with light exposure from 6:00 a.m. to 6:00 p.m.), and a background noise level of $40 \pm 10 \mathrm{~dB}$. Temperature was maintained at $20 \pm 3^{\circ} \mathrm{C}$. Prior to commencement of the experiment, all rabbits were acclimatized to these conditions for one week.

\section{Drugs and reagents}

Adriamycin hydrochloride (doxorubicin hydrochloride) was purchased from Shenzhen Main Luck Pharmaceutical Inc. (Shenzhen, China), while enalapril tablets were obtained from Shandong Cisen Pharmaceutical Ltd. (Jining, China). Antibodies against ERK1/2, phosphorylated ERK1/2 (p-ERK1/2), ERK5, and p-ERK5 were procured from Santa Cruz Biotechnology, Inc. (Dallas, TX, USA). Inhibitor PD98059 was acquired from Selleck Chemicals, Inc. (Houston, TX, USA).

\section{Methods}

\section{Experimental groups}

Forty New Zealand rabbits were randomly divided into the following eight groups, each comprising five animals: blank control, model control, PD98059 (PD) low-dose, PD high-dose, PD blank, dimethyl sulfoxide (DMSO) control, DMSO blank,and positive control.

\section{Establishment of abnormal ERK signaling pathway expression model}

Adriamycin hydrochloride for injection was diluted with saline solution to give a concentration of $1.0 \mathrm{mg} / \mathrm{mL}$, before being administered to rabbits via the marginal ear vein at a dose of 1.0 $\mathrm{mL} / \mathrm{kg}$, as previously described (Cai, 2014; Chen et al., 2014). Rabbits were injected twice a week, for four weeks.

\section{Drug administration}

Each group was injected with Adriamycin, with the exception of the blank control, PD blank, 
and DMSO blank groups. PD98059 was diluted with DMSO solution to a concentration of $1.0 \mathrm{mg} / \mathrm{mL}$, and from Day 1, rabbits in PD low-dose, PD high-dose,and PD blank groups were subcutaneously (sc) injected with doses of 20,40 , and $40 \mu \mathrm{g} \cdot \mathrm{kg}^{-1} \cdot$ day $^{-1}$, respectively. Rabbits in the DMSO control and DMSO blank groups were given DMSO solution at a dose of $40 \mu \mathrm{L} \cdot \mathrm{kg}^{-1} \cdot \mathrm{day}^{-1} \mathrm{sc}$. Animals of the positive control group were orally administered enalapril tablets at a dose of $5 \mathrm{mg} \cdot \mathrm{kg}^{-1} \cdot \mathrm{day}^{-1}$ (Yu et al., 2005). Enalapril causes hypertrophied myocardium. Those in the blank control group were treated with the same dose of saline solution. Each treatment was carried out continuously over the course of four weeks.

\section{Sample collection and detection}

\section{Extraction of total protein from myocardial tissues}

To measure the changes in each rabbit model induced by Adriamycin, animals from each group were euthanized and ventricular myocardial tissues were immediately placed in liquid nitrogen for cryopreservation. The frozen tissues were subsequently transferred to centrifuge tubes prior to being accurately weighed and homogenized in tissue lysis buffercontaining $50 \mathrm{mMTris}-\mathrm{HCl}$ and $50 \mathrm{mM} \mathrm{NaCl}$, based on a 1:8 weight:volume ratio, using a mechanical homogenizer. After being incubated in an ice bath and vortexed, the myocardial homogenates were left for $1 \mathrm{~h}$ at room temperature and centrifuged at $18,000 \mathrm{~g}$ for $30 \mathrm{~min}$ at $4^{\circ} \mathrm{C}$ to enable removal of debris. The supernatants, representing total myocardial tissue protein samples, were then subjected to a Bradford protein assay to measure protein concentration, and stored at $-70^{\circ} \mathrm{C}$ for later use.

\section{Western blot}

Myocardial total protein $(50 \mu \mathrm{g})$ was prepared in $2 \mathrm{X}$ sodium dodecyl sulfate (SDS) sample buffer $(0.1 \mathrm{M}$ Tris, pH 6.8, $0.2 \mathrm{M}$ dithiothreitol, 4\% SDS, $20 \%$ glycerol, and $0.02 \%$ bromophenol blue), before being denatured at $100^{\circ} \mathrm{C}$ for $8 \mathrm{~min}$ and loaded onto a polyacrylamidegel $(10 \%$ acrylamide). Constant-current electrophoresis was performed at $6 \mathrm{~mA} / \mathrm{gel}$ for $15 \mathrm{~min}$, and then $32 \mathrm{~mA} /$ gel, until the tracking dye reached the anode end. For electroblotting, the current was set to 0.8 $\mathrm{A} / \mathrm{cm}^{2}$. The proteins were transferred onto a nitrocellulose membrane for $2 \mathrm{~h}$. Following this, the membrane was blocked for $2 \mathrm{~h}$ at room temperature with Tris-buffered saline/Tween 20 (TBST) (Aladin Ltd., Shanghai, China) containing 3\% skim milk. The membrane was then placed in a hybridization bag and incubated with ERK1/2, p-ERK1/2, ERK5, and p-ERK5 antibodies on separate occasions at $4^{\circ} \mathrm{C}$ overnight. Three 10-min rinses were performed using TBST before placing the membrane into another hybridization bag to be exposed to the enzyme-conjugated secondary antibody for $2 \mathrm{~h}$ at room temperature. A further three 10-min TBST rinses were carried out, after whichthe blots were visualized with enhanced chemiluminescence reagents (BD Biosciences, USA) and scanned at $300 \mathrm{dpi}$. The images were saved for analysis.

\section{Measurement of myocardial ERK1/2 and ERK5 mRNA expression}

Total RNA was extracted from left ventricular myocardial tissues by the TRIzol one-step method, using TRIquick Reagent (Solarbio Science \& Technology CO., Ltd., Beijing, China), and an ultraviolet spectrophotometer was used for quantification and purity measurements. mRNA expression was determined by reverse transcription-polymerase chain reaction analysis (RT-PCR), following the manufacturer protocol. The forward and reverse primers were designed according to previ- 
ously published $E R K 1 / 2$, ERK5, and GAPDH rabbit (Oryctolagus cuniculus) gene sequences, and wereas follows: 5'-CCTGGAAGCCATGAGGGATGTCTAC-3' (F) and 5'-GCAGATGTGGTCGTTG CTTAGTTGC-3' (R) for ERK1/2 (based on Ref Seq accession No. XM_008257945); 5'-CCCCTCCCCCTTCTACATCAGAGTC-3' (F) and 5'-GTCAGCCACACCCATGTCAAAAGAC-3' (R) for ERK5 (based on RefSeq accession No. XM_008250339); and 5'-TTCAACAGTGCCACCCA CTCCTCTA-3' (F) and 5'-CCCTGTTGCTGTAGCCAAATTCGTT-3' (R) for GAPDH (based on RefSeq accession No. NM_001082253).

\section{Statistical analysis}

Data are reported as means \pm SD. All data were tested for normality and processed using SPSS17.0 statistical analysis software (SPSS Inc., Chicago, IL, USA). Significant differences between experimental groups were determined by a one-way analysis of variance. The least significant difference test was employed where equal variances were assumed. In other cases, the Tamhane $\mathrm{T} 2$ test was used. $\mathrm{P}<0.05$ was considered to be statistically significant.

\section{RESULTS}

\section{Assessment of animals}

As shown in Tables 1 and 2, with the exception of the DMSO control group, which included only four experimental animals by the completion of the experiment, each group comprised five rabbits throughout the trial. One DMSO control rabbit died before the end of the investigation, possibly due to acute poisoning induced by high-speed injection of Adriamycin.

Table 1. Myocardial ERK1/2 expression in each group measured after completion of the experiment (mean \pm SD).

\begin{tabular}{lcccc}
\hline Group & $\mathrm{N}$ & ERK1/2/Actin1 $(\mathrm{nM} / \mathrm{g})$ & $\mathrm{p}$-ERK1/2/Actin2 $(\mathrm{nM} / \mathrm{g})$ & ERK1/2/GAPDH mRNA (nM/g) \\
\hline Blank control & 5 & $0.45 \pm 0.08$ & $0.41 \pm 0.06$ & $1.18 \pm 0.18$ \\
Model control & 5 & $0.45 \pm 0.09^{\mathrm{a}}$ & $0.97 \pm 0.08^{\mathrm{b}}$ & $1.20 \pm 0.19^{\mathrm{a}}$ \\
PD low-dose & 5 & $0.44 \pm 0.09^{\mathrm{a}}$ & $0.65 \pm 0.07^{\mathrm{bc}}$ & $1.21 \pm 0.17^{\mathrm{a}}$ \\
PD high-dose & 5 & $0.46 \pm 0.08^{\mathrm{a}}$ & $0.53 \pm 0.06^{\mathrm{bcef}}$ & $1.18 \pm 0.19^{\mathrm{a}}$ \\
PD blank & 5 & $0.45 \pm 0.07^{\mathrm{a}}$ & $0.13 \pm 0.03^{\text {bce }}$ & $1.19 \pm 0.18^{\mathrm{a}}$ \\
DMSO control & 4 & $0.45 \pm 0.08^{\mathrm{a}}$ & $0.95 \pm 0.08^{\text {bde }}$ & $1.20 \pm 0.19^{\mathrm{a}}$ \\
DMSO blank & 5 & $0.47 \pm 0.09^{\mathrm{a}}$ & $0.43 \pm 0.07^{\text {acef }}$ & $1.19 \pm 0.17^{\mathrm{a}}$ \\
Positive control & 5 & $0.45 \pm 0.06^{\mathrm{a}}$ & $0.82 \pm 0.08^{\text {bce }}$ & $1.18 \pm 0.19^{\mathrm{a}}$ \\
\hline
\end{tabular}

PD = PD98059; DMSO = dimethyl sulfoxide. Compared with blank control group: aP > 0.05, bP < 0.05; compared with model control group: ${ }^{\mathrm{C}}<0.05$, ${ }^{\mathrm{P}}>0.05$; compared with $\mathrm{PD}$ low-dose group: $\mathrm{P}<0.05$; compared with $\mathrm{PD}$ blank group: ${ }^{\mathrm{f}} \mathrm{P}<0.05$.

Table 2. Myocardial ERK5 expression in each group measured after completion of the experiment (mean \pm SD).

\begin{tabular}{llccc}
\hline Group & $\mathrm{N}$ & ERK5/Actin3 $(\mathrm{nM} / \mathrm{g})$ & $\mathrm{p}$-ERK5/Actin4 $(\mathrm{nM} / \mathrm{g})$ & ERK5/GAPDH mRNA $(\mathrm{nM} / \mathrm{g})$ \\
\hline Blank control & 5 & $0.05 \pm 0.01$ & $0.81 \pm 0.04$ & $0.43 \pm 0.01$ \\
Model control & 5 & $0.05 \pm 0.01^{\mathrm{a}}$ & $0.52 \pm 0.03^{\mathrm{b}}$ & $0.42 \pm 0.01^{\mathrm{a}}$ \\
PD low-dose & 5 & $0.05 \pm 0.01^{\mathrm{a}}$ & $0.68 \pm 0.03^{\mathrm{bc}}$ & $0.43 \pm 0.02^{\mathrm{a}}$ \\
PD high-dose & 5 & $0.06 \pm 0.02^{\mathrm{a}}$ & $0.76 \pm 0.04^{\text {bce }}$ & $0.43 \pm 0.01^{\mathrm{a}}$ \\
PD blank & 5 & $0.05 \pm 0.01^{\mathrm{a}}$ & $0.53 \pm 0.03^{\text {bde }}$ & $0.42 \pm 0.01^{\mathrm{a}}$ \\
DMSO control & 4 & $0.06 \pm 0.02^{\mathrm{a}}$ & $0.82 \pm 0.04^{\text {ace }}$ & $0.43 \pm 0.02^{\mathrm{a}}$ \\
DMSO blank & 5 & $0.05 \pm 0.01^{\mathrm{a}}$ & $0.60 \pm 0.03^{\text {bce }}$ & $0.42 \pm 0.02^{\mathrm{a}}$ \\
Positive control & 5 & $0.05 \pm 0.01^{\mathrm{a}}$ & $0.43 \pm 0.01^{\mathrm{a}}$ \\
\hline
\end{tabular}

$\mathrm{PD}=$ PD98059; DMSO = dimethyl sulfoxide. Compared with blank control group: ${ }^{\mathrm{P}} \mathrm{P}>0.05,{ }^{\mathrm{b}} \mathrm{P}<0.05$; compared with model control group: ${ }^{\mathrm{P}}<0.05$, ${ }^{\mathrm{d}} \mathrm{P}>0.05$; compared with $\mathrm{PD}$ low-dose group: ${ }^{\mathrm{e}} \mathrm{P}<0.05$; compared with $\mathrm{PD}$ blank group: ${ }^{\mathrm{f}} \mathrm{P}<0.05$. 


\section{Post-experiment myocardial ERK1/2 expression}

Myocardial ERK1/2 expression in the experimental rabbits is shown in Table 1 and Figure 1. After inducing myocardial changes with injections of Adriamycin, ERK1/2 phosphorylation increased, as determined by western blot analysis (Figure $1 \mathrm{~A}$ ), while the total amount of ERK1/2 protein was unaltered. Excluding the blank control, PD blank, and DMSO blank groups, strong p-ERK1/2 signals were evident in all treatment categories. As shown in Table 1, the expression of $\mathrm{p}$-ERK1/2 in each group treated with Adriamycin significantly differed from that of the blank control group $(P<0.05)$. Moreover, in the DMSO blank and control groups, $p-E R K 1 / 2$ levels were essentially no different from those of the blank and model control groups, respectively $(P>0.05)$, indicating that exposure to DMSO did not interfere with the experimental outcome. Compared to their respective controls, the model control group showed a larger increase in p-ERK1/2 expression $(P<0.05)$ than the $P D$ low-dose, $P D$ high-dose, and positive control groups $(P<0.05)$. The $P D$ high-dose group demonstrated the smallest increase $(P<0.05)$. No significant differences in non-phosphorylated ERK1/2 protein or mRNA levels were observed between the groups $(P>$ 0.05). Figure $1 \mathrm{~B}$ and the data presented in Table 1 demonstrate that $E R K 1 / 2 \mathrm{mRNA}$ levels were the same in the PD98059 and blank control groups $(P>0.05)$, indicating that this inhibitor cannot suppress the expression of ERK1/2 transcripts, irrespective of dose.

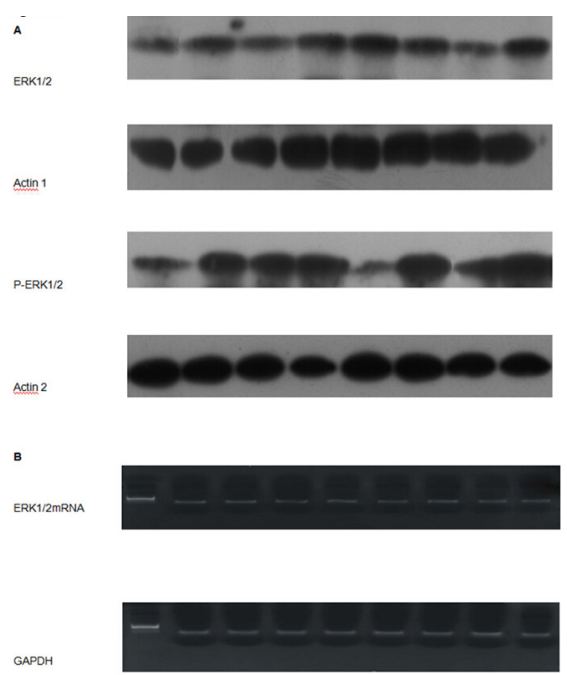

Figure 1. Myocardial ERK1/2 expression in each group. A. Western blot analysis of phosphorylated (p-ERK1/2) and non-phosphorylated ERK1/2 protein, showing representative bands for each group. B. Reverse transcriptionpolymerase chain reaction analysis of ERK1/2 and GAPDH mRNA expression. Samples are shown in the following order (from left to right lanes): blank control, model control, PD98059 (PD) low-dose, PD high-dose, PD blank, dimethyl sulfoxide (DMSO) control, DMSO blank, and positive control groups.

\section{Post-experiment myocardial ERK5 expression}

Myocardial ERK5 expression in the experimental rabbits is shown in Table 2 and Figure 2. Our western blot revealed strong p-ERK5 bands for each group. In addition, ERK5 phosphorylation was found to be increased in rabbits treated with Adriamycin (Figure 2A). The data reported in 
Table 2 demonstrate that expression of p-ERK5 in each Adriamycin-treated group was significantly different from that of the blank control group $(P<0.05)$. Moreover, $p-E R K 5$ levels in the DMSO blank and control groupsdid not significantly differ from those of the blank and model control groups $(P>0.05)$, respectively. However, PD98059 significantly decreased the extent of ERK5 phosphorylation in the PD low- and high-dose groups, indicating that this molecule is capable of suppressing p-ERK5 in rabbits. p-ERK5 expression was reduced to a greater degreein the model control group $(P<0.05)$ than in the PD low-dose, PD high-dose, and positive control groups $(P<0.05)$. The level of $p$-ERK5 slightly decreased in the PD high-dose group $(P<0.05)$. No significant differences in non-phosphorylated ERK5 protein or mRNA levels were detected between the groups $(P>0.05)$. As shown in Figure 2B and Table 2, the level of ERK5 mRNA was similar in all PD98059-treated animals compared with the blank control groups $(P>0.05)$, indicating that this compound is unable inhibit the expression of ERK5 mRNA, regardless of dose.

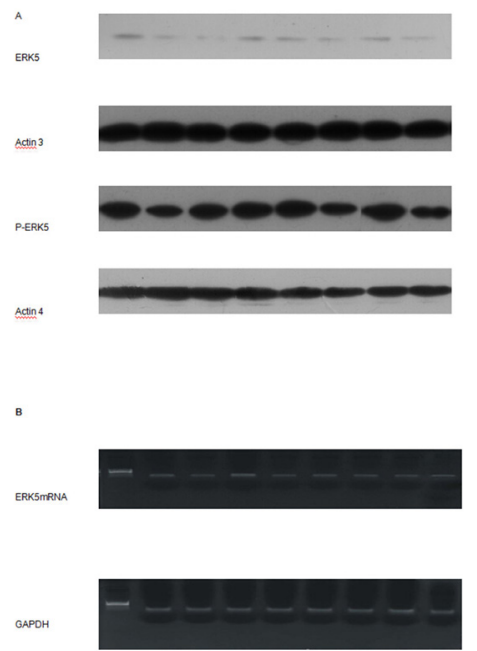

Figure 2. Myocardial ERK5 expression in each group. A. Western blot analysis of phosphorylated ( $p$-ERK5) and nonphosphorylated ERK5 protein, showing representative bands for each group and normalized densitometric ratios of ERK5 to actin3 and p-ERK1/2 to actin 4. B. Reverse transcription-polymerase chain reaction analysis of ERK5 and GAPDH mRNA expression. Samples are shown in the following order (from left to right lanes): blank control, model control, PD98059 (PD) low-dose, PD high-dose, PD blank, dimethyl sulfoxide (DMSO) control, DMSO blank, and positive control groups.

\section{DISCUSSION}

MAPKs, as threonine protein kinases, are involved in many diseases and activated by a diverse range of extracellular signals or stimuli. As described in other articles in this issue, structural and biophysical aspects of these enzymes have been studied in great detail. The four best characterized MAPK pathways, ERK1/2, JNK, p38, and ERK5, have been implicated in various aspects of cardiac regulation, from development to pathological remodeling (Wang, 2007). Both the ERK1/2 and ERK5 pathway are sensitive to PD98059, a well-known inhibitor of ERK signaling.

Most in vivo experiments involving PD98059 use high-dose intravenous or intraperitoneal injections, resulting in the death of experimental animals within 48 to $72 \mathrm{~h}$, and influencing any index of effect used (Di Paola et al., 2010). As this limits their value in long-term experiments, these 
methods should be employed for the investigation of pathological processes and the intervention effect of PD98059 in chronic diseases.

Adriamycin is one of the most effective chemotherapeutic agents against a variety of cancers, but causes chronic side effects, including development of cardiomyopathy and ultimately, congestive heart failure (Lefrak at al., 1973). In this experiment, Adriamycin-induced myocardiopathy was used as an abnormal expression model of ERK signaling, robustly activating both ERK $1 / 2$ and ERK5. Such myocardial models generated by the application of this drugmay prove to be valuable in long-term investigations. Owing to the death of one experimental animal in the DMSO control group, subsequent studies should avoid the high-speed injection of Adriamycin.

The present study, in which we explored appropriate methods for the use of PD98059 in long-term in vivo experiments, should provide a theoretical and practical basis for related fundamental research.

\section{Conflicts of interest}

The authors declare no conflict of interest.

\section{ACKNOWLEDGMENTS}

Research supported by the National Natural Science Foundation of China (\#81173213) and the Scientific Fund of Hunan Provincial Education Department (\#14A112).

\section{REFERENCES}

Cai H (2014). A clinical research on chronic heart failure patients and the effects of Wenyangzhenshuai granule on extracellular signal-regulated kinase 1/2 expression in the myocardial tissue in a rabbit model of chronic heart failure. Doctoral thesis. Hunan University of Traditional Chinese Medicine, Changsha.

Chen XY, Cai HZ, Shi W, Chen QY, et al. (2014). Effects of WenyangZhenshuai granule on the expression of extracellular signal-regulated kinase 5 of the myocardial tissue in rabbit model with chronic heart failure induced by adriamycin. $J$. Hunan Univ. Chin. Med. 34: 14-18.

Chen S, Yang Y, Feng H, Wang H, et al. (2014). Baicalein inhibits interleukin-1 $\beta$-induced proliferation of human rheumatoid arthritis fibroblast-like synoviocytes. Inflammation 37: 163-169.

Di Paola R, Galuppo M, Mazzon E, Paterniti I, et al. (2010). PD98059, a specific MAP kinase inhibitor, attenuates multiple organ dysfunction syndrome/failure (MODS) induced by zymosan in mice. Pharmacol. Res. 61: 175-187.

Dudley DT, Pang L, Decker SJ, Bridges AJ, et al. (1995). A synthetic inhibitor of the mitogen-activated protein kinase cascade. Proc. Natl. Acad. Sci. U.S.A. 92: 7686-7689.

Gregg J and Fraizer G (2011). Transcriptional regulation of EGR1 by EGF and the ERK signaling pathway in prostate cancer cells. Genes Cancer 2: 900-909.

Hayashi M, Kim SW, Imanaka-Yoshida K, Yoshida T, et al. (2004). Targeted deletion of BMK1/ERK5 in adult mice perturbs vascular integrity and leads to endothelial failure. J. Clin. Invest. 113: 1138-1148.

Kato H, Nishida K, Yoshida A, Takada I, et al. (2003). Effect of NOS2 gene deficiency on the development of autoantibody mediated arthritis and subsequent articular cartilage degeneration. J. Rheumatol. 30: 247-255.

Lefrak EA, Pitha J, Rosenheim S and Gottleib JA (1973). A clinicopathologic analysis of adriamycin cardiotoxicity. Cancer 32 : 302-314.

Li L, Chen W, Zhu Y, Wang X, et al. (2014). Caspase recruitment domain 6 protects against cardiac hypertrophy in response to pressure overload. Hypertension 64: 94-102.

Nishimoto S and Nishida E (2006). MAPK signaling: ERK5 versus ERK1/2. EMBO Rep. 7: 782-786.

Regan CP, Li W, Boucher DM, Spatz S, et al. (2002). ERK5 null mice display multiple extraembryonic vascular and embryonic cardiovascular defects. Proc. Natl. Acad. Sci. U.S.A. 99: 9248-9253.

Wang Y (2007). Mitogen-activated protein kinases in heart development and diseases. Circulation 116: $1413-1423$. 
Yasaka A and Hayashida W (2001). Alterations of load-induced p38MAP kinase activation in failing rat hearts. Biochem. Biophys. Res. Commun. 285: 503-507.

Yu HZ, Yang Q and Deng JL (2005). Effect of electrical remodeling in hypertrophied myocardium caused by enalapril and its mechanisms. Zhonghua Xin Xue Guan Bing Za Zhi 33: 565-567.

Yuan LQ, Zhu JH, Wang HW, Liang QH, et al. (2011). RANKL is a downstream mediator for insulin-induced osteoblastic differentiation of vascular smooth muscle cells. PLoS One 6: e29037.

Zhang W and Liu HT (2002). MAPK signal pathways in the regulation of cell proliferation in mammalian cells. Cell Res. 12: 9-18.

Zhang Y, Yu JB, Luo XQ, Gong LR, et al. (2014). Effect of ERK1/2 signaling pathway in electro-acupuncture mediated upregulation of heme oxygenase-1 in lungs of rabbits with endotoxic shock. Med. Sci. Monit. 20: 1452-1460.

Zhou G, Bao ZQ and Dixon JE (1995). Components of a new human protein kinase signal transduction pathway. J. Biol. Chem. 270: $12665-12669$ 